\title{
Plasma urate, lung function and chronic obstructive pulmonary disease: a Mendelian randomisation study in 114979 individuals from the general population
}

\author{
Camilla J Kobylecki, ${ }^{1,2}$ Signe Vedel-Krogh, ${ }^{1,2}$ Shoaib Afzal, ${ }^{1,2}$ Sune F Nielsen, ${ }^{1,2}$ \\ Børge G Nordestgaard ${ }^{1,2}$
}

\begin{abstract}
- Additional material is published online only. To view please visit the journal online (http://dx.doi.org/10.1136/ thoraxjnl-2017-210273).

${ }^{1}$ Department of Clinical Biochemistry, Herlev and Gentofte Hospital, Copenhagen University Hospital, Herlev, Denmark

2Department of Clinical Medicine, Faculty of Health and Medical Sciences, University of Copenhagen, Copenhagen, Denmark
\end{abstract}

\section{Correspondence to Professor Børge \\ $G$ Nordestgaard, Department of Clinical Biochemistry, Herlev and Gentofte Hospital, Copenhagen University Hospital, Herlev DK- 2730, Denmark: \\ Boerge.Nordestgaard@regionh. $\mathrm{dk}$}

Received 17 March 2017 Revised 25 October 2017 Accepted 6 November 2017 Published Online First 29 November 2017

\section{Linked}

- http://dx.doi.org/10.1136/ thoraxjnl-2017-211230

Check for updates

To cite: Kobylecki CJ, VedelKrogh S, Afzal S, et al. Thorax 2018:73:748-757.

\begin{abstract}
Background Urate is a strong antioxidant in plasma and may protect against lung function impairment. We tested the hypothesis that high plasma urate is causally associated with better lung function and low risk of respiratory symptoms and COPD.
\end{abstract}

Methods We measured lung function and plasma urate in 114979 individuals from the Copenhagen City Heart Study and the Copenhagen General Population Study and genotyped for SLC2A9 rs7442295 and ABCG2 rs2231142 variants, previously associated with high plasma urate, in 110152 individuals.

Results In the two studies combined, multivariableadjusted $100 \mu \mathrm{mol} / \mathrm{L}$ higher plasma urate was associated with $-1.54 \%(95 \% \mathrm{Cl}-1.67$ to -1.40$)$ lower $\mathrm{FEV}_{1} \%$ predicted and $-1.57 \%(95 \% \mathrm{Cl}-1.69$ to -1.44$)$ lower FVC \% predicted observationally; the corresponding estimates for genetically determined $100 \mu \mathrm{mol} / \mathrm{L}$ higher plasma urate were $-0.46 \%(95 \% \mathrm{Cl}-1.17$ to 0.25$)$ and $-0.40 \%(95 \% \mathrm{Cl}-1.03$ to 0.23$)$. High plasma urate was also associated with higher risk of respiratory symptoms; however, genetically determined high plasma urate was not associated with respiratory symptoms. Finally, we identified 14151 individuals with COPD and found ORs of 1.08 (95\% Cl 1.06 to 1.11$)$ for COPD observationally and 1.01 (95\% Cl 0.88 to 1.15$)$ genetically per 100 $\mu \mathrm{mol} / \mathrm{L}$ higher plasma urate.

Conclusion High plasma urate was associated with worse lung function and higher risk of respiratory symptoms and COPD in observational analyses; however, genetically high plasma urate was not associated with any of these outcomes. Thus, our data do not support a direct causal relationship.

\section{INTRODUCTION}

Urate is the final breakdown product of purines and is present in the epithelial lining fluid of the respiratory tract and in plasma. ${ }^{12}$ Urate is considered to be the main contributor to the antioxidant capacity of plasma, ${ }^{3}$ and may as such protect the lung from oxidative damage. ${ }^{45}$ Oxidative damage from reactive oxygen species (ROS) and free radicals, derived from normal cell metabolism or from exogenous sources such as air pollutants and cigarette smoke, ${ }^{6}$ can occur through numerous pathways. Through oxidation of different tissue components, that is, cells or interstitial matrix, ROS can induce activation of cellular stress signalling pathways, ${ }^{7}$ macrophage accumulation, ${ }^{8}$ inactivation of alpha 1 -antitrypsin, ${ }^{9}$

\section{Key messages}

What is the key question?

- Are high plasma urate levels causally associated with lung function, respiratory symptoms and COPD?

What is the bottom line?

- High plasma urate levels were associated with low lung function and high risk of respiratory symptoms and COPD; however, genetically high plasma urate was not.

Why read on?

- This is the first study to assess plasma urate per se as a causal risk factor for low lung function using a Mendelian randomisation design.

and initiate inflammation ${ }^{10-12}$ or airway remodelling. ${ }^{13}$ Thus, it seems plausible that antioxidants such as urate play a role in maintaining normal lung function. However, observational studies have shown conflicting results, as some have found high plasma urate to be associated with low lung function whereas others have found high plasma urate associated with lower incidence of COPD. ${ }^{14-18}$ In the present study, we used a novel approach to address the question of the role of plasma urate in lung function and COPD, the Mendelian randomisation design.

Mendelian randomisation refers to the random assortment of genes at the time of gamete formation, which allows for a genetic variant to be used to study risk of disease, largely free of confounding (as genotypes are randomly distributed), and reverse causation (a genotype cannot be altered by disease). ${ }^{19}$ Various genes have been associated with plasma urate; however, variation in SLC2A9 is the major genetic determinant of plasma urate. ${ }^{20}$ SLC2A9 encodes the GLUT9 urate transporter, involved in renal urate reabsorption, ${ }^{20-22}$ and the SLC2A9 rs7442295 variant is robustly associated with plasma urate levels. ${ }^{23}{ }^{24}$ Likewise, variations in the $A B C G 2$ gene, encoding $A B C G 2$, a secretory urate transporter located in the proximal renal tubules, have been robustly associated with plasma urate; one such variant of high prevalence in Europeans is the loss-of-function mutation rs $2231142 .{ }^{25}$ In this study, we applied the SLC2A9 


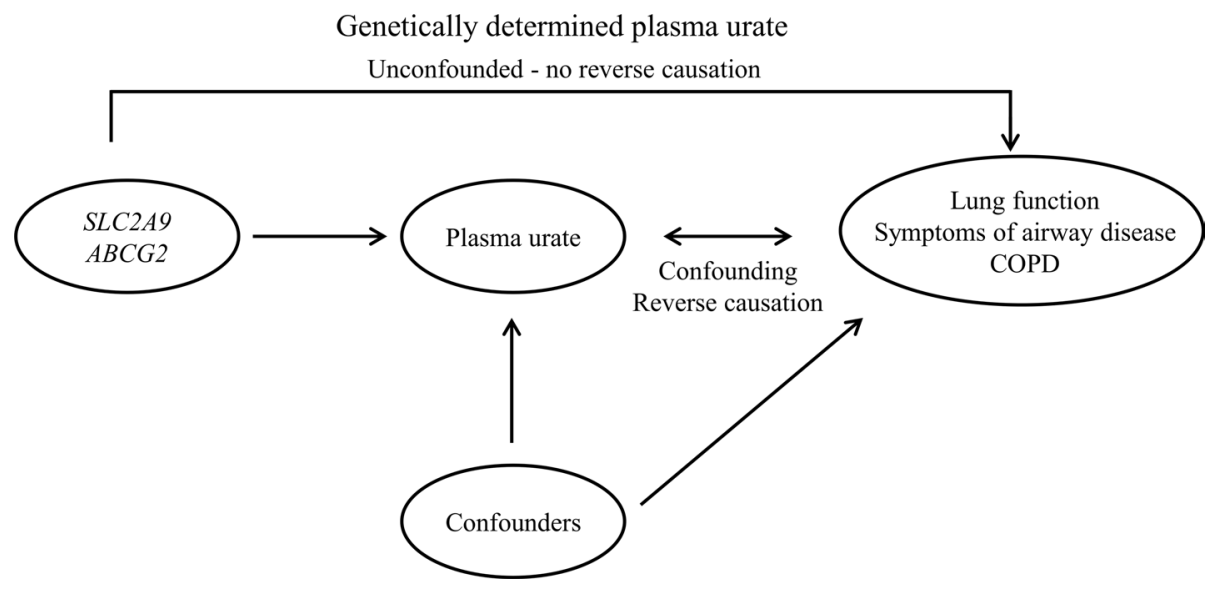

Figure 1 Study design. Outline of study design with possible associations between SLC2A9 rs7442295 and ABCG2 rs2231142 and plasma urate, between plasma urate and lung function, respiratory symptoms and COPD, and between measured and unmeasured confounders and plasma urate and outcomes. The direct associations of SLC2A9 and $A B G C 2$ with lung function, respiratory symptoms and COPD represent the Mendelian randomisation part.

rs7442295 and ABCG2 rs2231142 in a Mendelian randomisation design to assess the potential causal effect of high plasma urate on lung function, respiratory symptoms and risk of COPD.

First, we tested if high plasma urate was associated with high $\mathrm{FEV}_{1} \%$ predicted, FVC \% predicted, and low risk of respiratory symptoms and COPD in 114979 individuals from the Copenhagen General Population Study (CGPS) and the Copenhagen City Heart Study (CCHS). Second, we tested if a combined allele score was associated with high plasma urate in the same populations. Finally, we used this combined allele score to test if genetically determined high levels of urate were associated with high lung function and low risk of respiratory symptoms and COPD in order to infer causality. The study design is outlined in figure 1.

\section{METHODS}

\section{Study population}

We used the CCHS, initiated in 1976-1978 with follow-up examinations in 1981-1983, 1991-1994 and 2001-2003, and the CGPS, initiated in 2003 with ongoing enrolment. ${ }^{26-28}$ DNA was available in the 1991-1994 and 2001-2003 examinations of the CCHS and in the CGPS. For both studies, individuals aged 20-100 years were invited randomly from the Danish Civil Registration System to complete a questionnaire, undergo physical examination and give blood for plasma measurements and DNA analyses. There was no overlap of individuals between the two studies. Participation rate was $61 \%$ and $50 \%$ in the CCHS 1991-1994 and 2001-2003 examinations, respectively, and $45 \%$ in the CGPS. The studies were approved by Herlev and Gentofte Hospital and Danish Ethical Committees, conducted according to the Declaration of Helsinki, and written informed consent was obtained from all participants. We only included individuals of Danish descent, giving a total of 9779 individuals from the CCHS and 105200 from the CGPS with plasma urate measurements; in the two studies, 9612 (CCHS) and 100540 individuals (CGPS), respectively, had been genotyped for both urate SLC2A9 rs7442295 and ABCG2 rs2231142.

\section{Covariates}

Baseline characteristics were recorded from a self-administered questionnaire, reviewed together with an examiner at the day of attendance, a physical examination, and from blood samples. Information on asthma, smoking, alcohol intake, physical activity, income, education, exposure to occupational dust and fumes, and intake of meat (only available in the CGPS) was self-reported. Cumulative tobacco consumption in packyears was 20 cigarettes or equivalent smoked per day for 1 year. Ever-smokers were current or former smokers. Body mass index (BMI) was calculated from measured weight in kilograms divided by measured height in metres squared. Pre-existing cardiovascular disease was defined as a diagnosis of ischaemic heart disease prior to examination (WHO International Classification of Diseases (ICD)-8 codes 410-414, and ICD-10 codes I20-I25), collected from the Danish National Patient Registry. If these data were not available (approximately 10000 individuals recruited after 2013 where follow-up ended), pre-existing cardiovascular disease was defined as self-reported hospitalisation due to myocardial infarction, myocardial infarction without hospitalisation, coronary artery bypass grafting or percutaneous coronary intervention.

\section{Outcomes}

In the CCHS and in the first 15000 participants of the CGPS, spirometry was performed with a Vitalograph (Maids Moreton, Buckinghamshire, UK), and in the remainder of CGPS with an EasyOne Spirometer (ndd Medical Technologies, Zurich, Switzerland). Only prebronchodilator measurements of $\mathrm{FEV}_{1}$ and $\mathrm{FVC}$ were available; these were measured with at least three sets of values, and two measurements differing by $<5 \%$ was a criterion for reproducibility. The highest measurements of $\mathrm{FEV}_{1}$ and FVC were used in the analyses. We calculated predicted values of $\mathrm{FEV}_{1}, \mathrm{FVC}$ and the $\mathrm{FEV}_{1} / \mathrm{FVC}$ ratio using the prediction formulas for a Danish population (CGPS) derived by Løkke et al. ${ }^{27}$ We defined COPD as $\mathrm{FEV}_{1} / \mathrm{FVC}$ below the lower limit of normal; the fifth percentile of a frequency distribution, using the prediction equation estimates derived from a healthy non-smoking reference population of similar sex. ${ }^{27}$ Those with self-reported asthma were excluded from the COPD analyses $(n=6809)$. All respiratory symptoms were self-reported. We dichotomised the modified Medical Research Council (mMRC) score, and defined $m M R C \geq 2$ if the participant reported more shortness of breath than peers, shortness of breath when walking in his/her own pace, or shortness of breath when getting dressed. Any dyspnoea was any report of breathlessness. Sputum 
Table 1 The association of baseline characteristics with urate tertiles, COPD and the combined allele score in the Copenhagen General Population Study (CGPS)

\begin{tabular}{|c|c|c|c|c|c|c|c|c|}
\hline & \multicolumn{3}{|l|}{ Urate tertiles } & \multirow[b]{2}{*}{$P$ for trend } & \multicolumn{2}{|l|}{ COPD } & \multirow[b]{2}{*}{$\mathbf{P}$} & \multirow{2}{*}{$\begin{array}{l}\text { Allele score } \\
\text { P for trend }\end{array}$} \\
\hline & First & Second & Third & & No & Yes & & \\
\hline No. of individuals & 38394 & 34427 & 32379 & $\mathrm{n}=105200$ & 86009 & 12957 & $n=98966$ & $\mathrm{n}=100540$ \\
\hline Plasma urate $(\mu \mathrm{mol} / \mathrm{L})$ & $230(200-280)$ & $290(260-350)$ & $390(330-430)$ & & $300(240-360)$ & $300(250-370)$ & $6 \times 10^{-25}$ & $<1 \times 10^{-300}$ \\
\hline Men, $n(\%)$ & $17089(45)$ & $15678(46)$ & $14599(45)$ & 0.33 & $38475(45)$ & $6418(50)$ & $2 \times 10^{-24}$ & 0.83 \\
\hline Age (years) & $55(46-65)$ & $58(48-67)$ & $62(52-70)$ & $<1 \times 10^{-300}$ & $57(48-67)$ & $63(53-71)$ & $<1 \times 10^{-300}$ & 0.41 \\
\hline Ever-smokers, n (\%) & 21352 (56) & 19956 (58) & $20111(62)$ & $2 \times 10^{-68}$ & $47932(56)$ & $9780(75)$ & $<1 \times 10^{-300}$ & 0.43 \\
\hline Cumulative tobacco consumption (pack-years) & $14(5-27)$ & $15(6-29)$ & $19(8-34)$ & $7 \times 10^{-168}$ & $14(5-27)$ & $25(13-40)$ & $<1 \times 10^{-300}$ & 0.97 \\
\hline Body mass index $\left(\mathrm{kg} / \mathrm{m}^{2}\right)$ & $24(22-27)$ & $26(23-28)$ & $27(25-30)$ & $<1 \times 10^{-300}$ & $26(23-28)$ & $25(23-28)$ & $4 \times 10^{-63}$ & 0.14 \\
\hline Alcohol consumption (g/week) & $85(36-168)$ & $96(48-180)$ & $108(48-192)$ & $4 \times 10^{-133}$ & $96(48-180)$ & $108(48-204)$ & $2 \times 10^{-29}$ & 0.68 \\
\hline High income, $n(\%)$ & $18534(48)$ & 15410 (45) & 11444 (35) & $4 \times 10^{-258}$ & 38449 (45) & 4417 (34) & $2 \times 10^{-114}$ & 0.41 \\
\hline High physical activity in leisure time, $n(\%)$ & $2907(8)$ & $2385(7)$ & $1775(6)$ & $3 \times 10^{-28}$ & $5811(7)$ & $810(6)$ & 0.03 & 0.67 \\
\hline High education, $\mathrm{n}(\%)$ & $19278(50)$ & $16126(47)$ & $12512(39)$ & $8 \times 10^{-205}$ & $39885(46)$ & $5258(41)$ & $5 \times 10^{-35}$ & 0.41 \\
\hline $\begin{array}{l}\text { Exposure to occupational dust and fumes, } \mathrm{n} \\
(\%)\end{array}$ & $3312(9)$ & $3325(10)$ & $3890(12)$ & $5 \times 10^{-50}$ & $7881(9)$ & $1681(13)$ & $1 \times 10^{-42}$ & 0.02 \\
\hline High intake of meat, $n(\%)$ & $4297(11)$ & $3950(11)$ & $3933(12)$ & $3 \times 10^{-32}$ & $9834(11)$ & $1588(12)$ & $3 \times 10^{-5}$ & 0.92 \\
\hline Pre-existing cardiovascular disease, $\mathrm{n}(\%)$ & $1628(4)$ & $1775(5)$ & $2788(9)$ & $1 \times 10^{-129}$ & $4672(5)$ & $1060(8)$ & $9 \times 10^{-36}$ & 0.71 \\
\hline Creatinine $(\mu \mathrm{mol} / \mathrm{L})$ & $77(69-85)$ & $80(72-89)$ & $84(75-94)$ & $<1 \times 10^{-300}$ & $80(72-89)$ & $79(71-89)$ & $8 \times 10^{-8}$ & 0.41 \\
\hline C-reactive protein (mg/L) & $1.2(0.76-1.8)$ & $1.4(0.93-2.2)$ & $1.7(1.2-3.1)$ & $<1 \times 10^{-300}$ & $1.4(0.92-2.2)$ & $1.5(0.95-2.6)$ & $2 \times 10^{-31}$ & 0.94 \\
\hline
\end{tabular}

Continuous variables are shown as median and IQR. Urate tertiles were assigned by sex. COPD was defined as lower limit of normal. Allele score: urate SLC2A9 rs7442295

variant+urate $A B C G 2$ rs 2331142 variant. Individuals with self-reported asthma ( $n=6234$ in the CGPS) are excluded from COPD analyses. $P$ for trend was calculated using Cuzick's non-parametric test for trend.

(>3 months) was sputum production for at least 3 consecutive months/year. Individuals were characterised as having any respiratory symptoms if they reported any of the following: dyspnoea, wheezing from any reason, sputum production $(>3$ months), cough and/or $m M R C \geq 2$. Use of asthma/bronchitis medication was self-reported.

\section{Laboratory analyses}

We genotyped for SLC2A9 rs7442295 and ABCG2 rs2231142 using a TaqMan-based assay (Applied Biosystems) with a call rate of $>99 \%$ after reruns. Genotyping was verified by DNA sequencing in at least 30 individuals and these were used as controls for the further genotyping. We used DNA extracted from leukocytes in peripheral blood using the Qiagen blood kit for DNA extraction. Plasma urate was measured using Konelab autoanalyser and a standard Konelab assay as supplied by the manufacturer (Thermo Fischer Scientific). For the assay, the coefficient of variation was $4 \%$, lower limit of detection was $5 \mu \mathrm{mol} / \mathrm{L}$, and the measurement range was $10-1200 \mu \mathrm{mol} / \mathrm{L}$. No values of plasma urate or genotypes were imputed. Plasma high-sensitive C-reactive protein and creatinine were measured with standard hospital assays.

\section{Statistical analyses}

We used Stata V.13.1. A $\chi^{2}$ test was used to evaluate Hardy-Weinberg equilibrium. $\mathrm{P}$ values for trends were estimated using Cuzick's non-parametric trend test. All reported P values were two sided. The data were $>98 \%$ complete and missing values for covariates were imputed according to age and sex that had no missing values. For this purpose, we used multivariate normal imputation for continuous variables and chained equation for categorical variables. However, if individuals with any missing data were excluded, results were similar to those presented. In order to maximise statistical power, we combined the CCHS and
CGPS and adjusted the analyses for study by including study as a standard variable.

To test if plasma urate was associated with lung function parameters, we used linear regression, either adjusted for sex and age, or multivariable adjusted, which was for age (continuous), sex, smoking status (categorical), cumulative smoking (in 10 groups), BMI (in 3 groups), alcohol intake (continuous), meat intake (in 5 groups, only in CGPS), income (6 groups), education (6 groups), physical activity during leisure time (4 groups), exposure to occupational dust and fumes (yes/no), pre-existing cardiovascular disease (yes/no), plasma creatinine (in 5 groups) and plasma C-reactive protein (continuous). Grouping of continuous variables was chosen a priori based on assumptions of the relationship between covariate and outcome as well as the exactness of the self-reported values. The assumptions of linearity, homoscedasticity and normality were tested graphically using plots of observed versus predicted values and plots of residuals versus predicted values or the observed exposure values. No major violations were observed.

To test if plasma urate was associated with COPD, we performed multivariable-adjusted, restricted cubic spline logistic regression in the two studies combined to obtain ORs and 95\% CIs. ${ }^{29}$ For this purpose, we used percentiles of plasma urate, assigned by sex, study, and year of measurement and adjusted for the same covariates as in the linear regression models. To obtain point estimates for plasma urate tertiles, we performed multivariable-adjusted logistic regression in the two studies separately and combined.

To test whether plasma urate was associated with symptoms of airway disease in the two studies separately, we used Cuzick's non-parametric trend test and multivariable-adjusted logistic regression. Multivariable adjustment was done as in the linear regression models, only here we also included three categories of $\mathrm{FEV}_{1}\left(\mathrm{FEV}_{1} \%\right.$ predicted $\geq 80 \%, \mathrm{FEV}_{1} \%$ predicted $<80 \%$ and $>50 \%$, and $\mathrm{FEV}_{1} \%$ predicted $\leq 50 \%$ ). 
To test if urate SLC2A9 rs7442295 and ABCG2 rs2231142 genotypes were associated with plasma urate, we performed linear regression in the two studies combined from which also the $F$-statistic (strength of the genotype as an instrument) and $\mathrm{R}^{2}$ (measure of variation explained by genotype) were obtained. We combined the SLC2A9 rs7442295 and ABCG2 rs2231142 in a urate-increasing allele score ranging from 0 to 4 urate-increasing alleles in order to maximise power.

Potential causal relationships between genetically high plasma urate and lung function, respiratory symptoms and COPD were assessed by instrumental variable (IV) analyses using the control

\section{Copenhagen General Population Study}

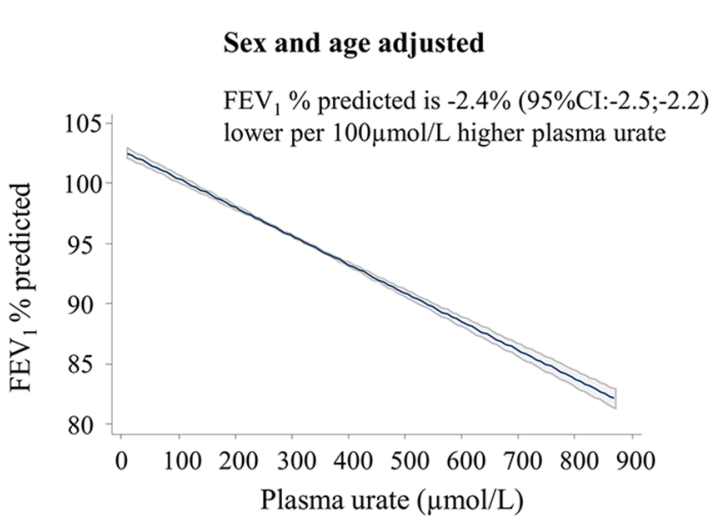

Multivariable adjusted

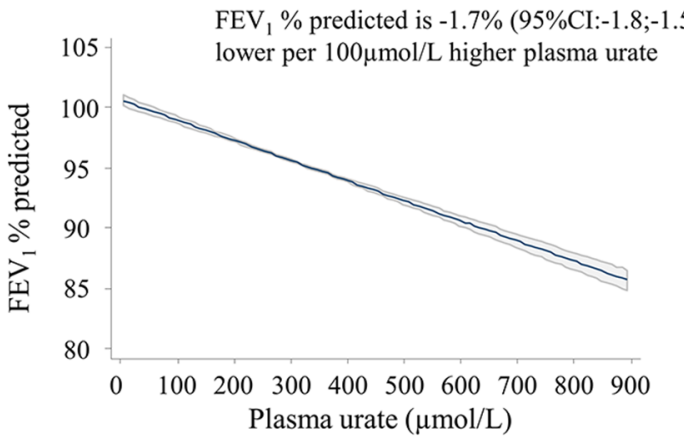

Stratified on smoking status

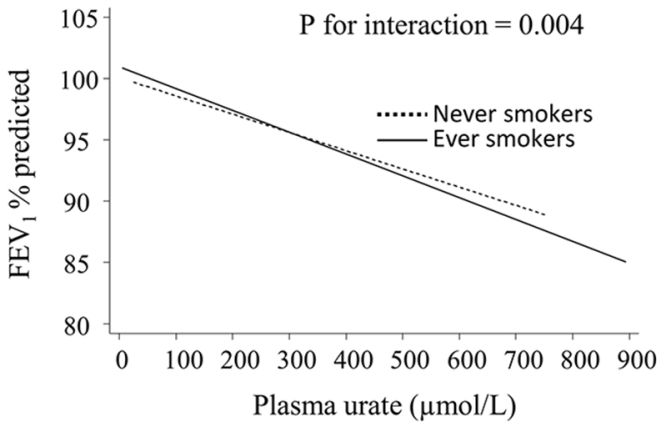

function IV estimator. ${ }^{30}$ This involves first a linear regression of plasma urate on the urate allele score on a continuous scale, generating predicted values for plasma urate, followed by either linear regression or logistic regression of the outcome on the predicted values of plasma urate including the estimated residuals from the linear regression in the first step. In these analyses, both incident and prevalent cases were included, as genotype is present from birth. We calculated SEs using 5000 bootstrap replications.

In sensitivity analyses, potential interactions of plasma urate and urate genotype with sex and ever/never-smokers were

\section{Copenhagen City Heart Study}

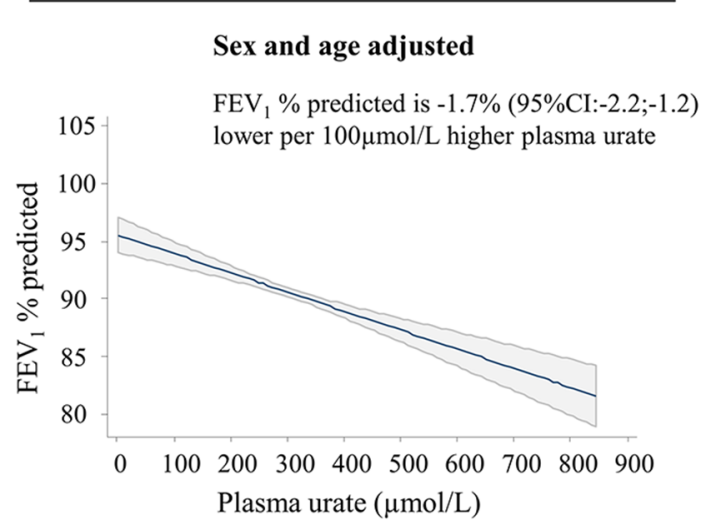

Multivariable adjusted
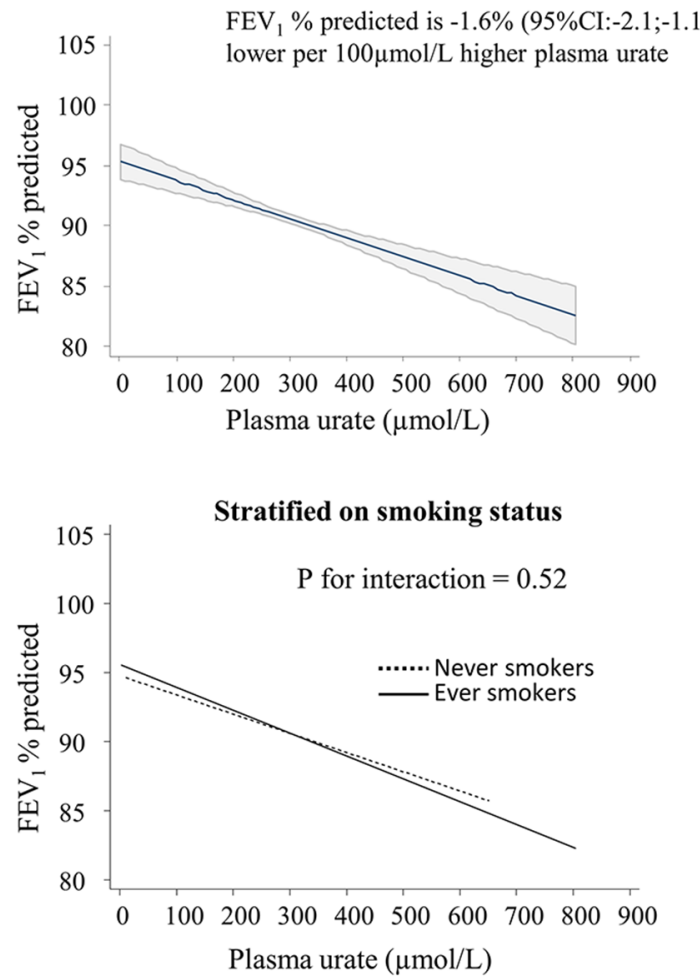

Figure 2 Regression of FEV 1 \% predicted on plasma urate in the Copenhagen General Population Study and the Copenhagen City Heart Study separately. In the Copenhagen General Population Study $(n=105200)$ the analysis was multivariable adjusted for age, sex, smoking status, cumulative smoking, body mass index, physical activity during leisure time, intake of meat, intake of alcohol, income, education, pre-existing cardiovascular disease, exposure to occupational dust and fumes, plasma creatinine and plasma C-reactive protein. In the Copenhagen City Heart Study ( $\mathrm{n}=9779$ ) the analysis was multivariable adjusted for all of the above except for meat intake, which was not available. Ever-smokers: former and current smokers combined. Regression values in top and middle panels are shown with 95\% Cls as shaded area around regression line; bottom panels show regression lines only. 
examined using a likelihood ratio test by introducing a two-factor interaction term in a model also including both factors. Furthermore, to explore if the role of plasma urate differed by COPD status, we performed the same analyses in the COPD subpopulation alone. Finally, we repeated the main analyses, first in complete cases, where all individuals with missing covariates were excluded, and second, in the combined study population, where analyses were adjusted for study by using it as a cluster variable instead of including study as a standard variable.

\section{RESULTS}

We identified 14151 individuals with COPD in the CGPS and the CCHS combined. Plasma urate and COPD were associated with most of the included confounders in both the CGPS and the CCHS (table 1 and online supplementary table 1). In contrast, neither SLC2A9 rs7442295, ABCG2 rs2231142 nor the combined allele score was associated with any of the confounders, and thus, can be used as largely unconfounded instruments for plasma urate (table 1 and online supplementary tables 2-5).

The genotype distributions did not differ from those expected under Hardy-Weinberg equilibrium (for SLC2A9 rs7442295 $\mathrm{P}=0.22$ and $\mathrm{P}=0.42$, for $A B C G 2$ rs2231142 $\mathrm{P}=0.15$ and $\mathrm{P}=0.11$ in the CGPS and the CCHS, respectively) and were not in linkage disequilibrium $\left(\mathrm{R}^{2}=0 \%\right)$.

\section{Plasma urate and lung function}

Multivariable-adjusted $100 \mu \mathrm{mol} / \mathrm{L}$ higher plasma urate was associated with a $-1.7 \%(95 \% \mathrm{CI}-1.8$ to -1.5$)$ lower $\mathrm{FEV}_{1}$ $\%$ predicted in the CGPS with a similar estimate in the CCHS (figure 2). For FVC $\%$ predicted, a multivariable-adjusted $100 \mu \mathrm{mol} / \mathrm{L}$ higher plasma urate was associated with a $-1.8 \%$ (95\% CI -1.9 to -1.7 ) lower FVC \% predicted in the CGPS, likewise with similar results in the CCHS (online supplementary figure 1). For $\mathrm{FEV}_{1} / \mathrm{FVC}$, a multivariable-adjusted $100 \mu \mathrm{mol} / \mathrm{L}$ higher plasma urate was associated with a $-0.09 \%$ (95\% CI -0.15 to -0.02 ) lower $\mathrm{FEV}_{1} / \mathrm{FVC}$ in the CGPS; however, no association with plasma urate was found in the CCHS (online supplementary figure 2).

\section{Plasma urate and COPD}

After multivariable adjustment, we found an OR of 1.05 (95\% CI 1.01 to 1.11 ) for COPD for those in the middle tertile and 1.08 (95\% CI 1.03 to 1.14 ) for those in the highest tertile compared with those in the lowest tertile of plasma urate (figure 3). In the two studies separately, we found the strongest association between plasma urate and risk of COPD in the CGPS (online supplementary figure 3 ); however, the sample size was approximately 10 times higher in the CGPS than in the CCHS.

\section{Plasma urate and symptoms of airway disease}

In the CGPS, after multivariable adjustment which included $\mathrm{FEV}_{1} \%$ predicted, individuals in the highest plasma urate tertile had an OR of 1.25 (95\% CI 1.20 to 1.30$)$ for any dyspnoea, 1.32 (95\% CI 1.23 to 1.41 ) for an mMRC score $\geq 2$ and 1.25 (95\% CI 1.20 to 1.29 ) for any respiratory symptoms compared with individuals in the lowest urate tertile; results were similar in the CCHS (table 2).

\section{Genetically high plasma urate, lung function, symptoms of airway disease and COPD}

In the two studies combined, a one-unit increase in the combined allele score was associated with 7\% (95\% CI 6.9 to 7.4 ) or
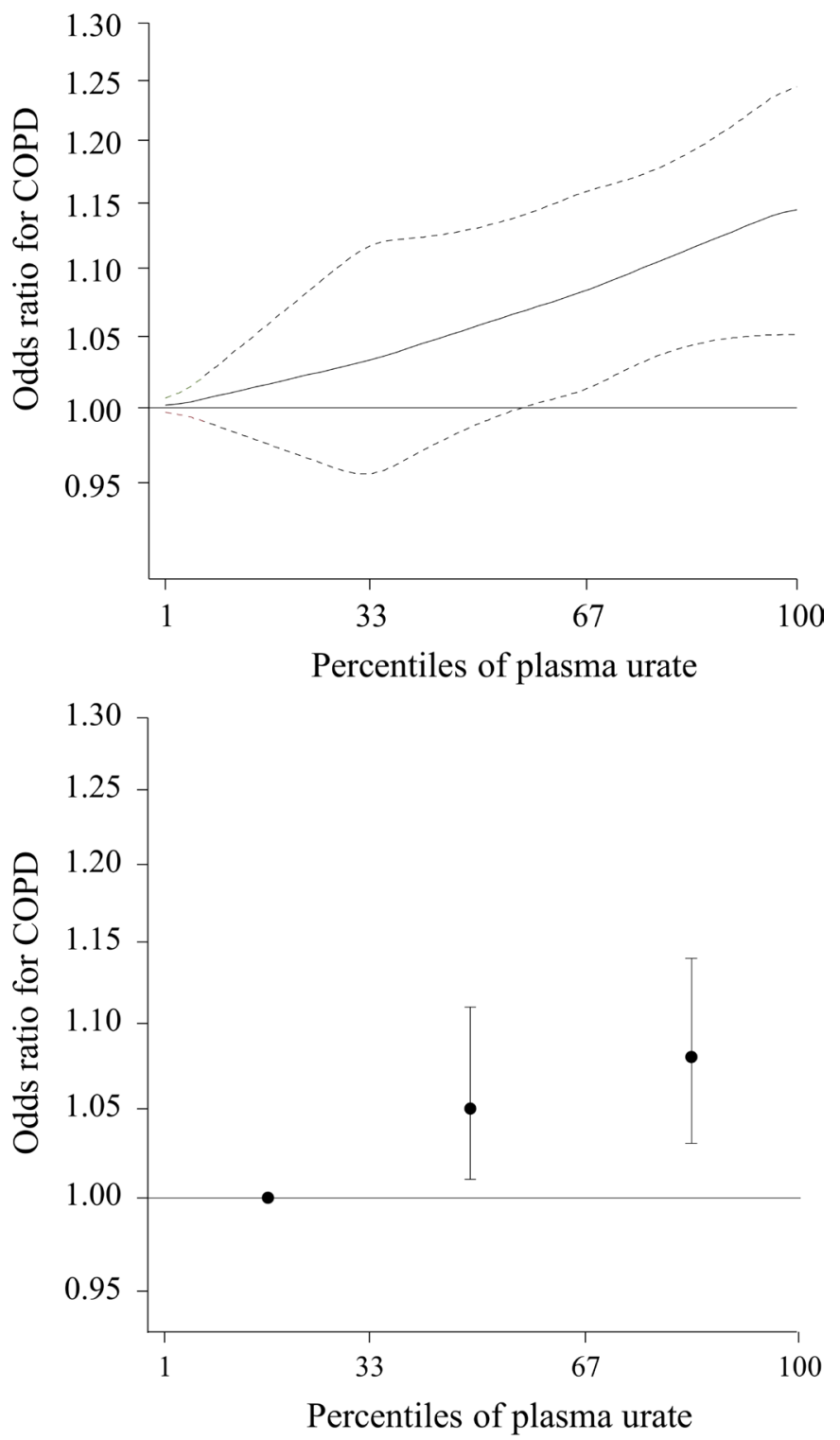

No. of

$\begin{array}{llll}\text { participants } & 39611 & 35622 & 32937 \\ \text { No. of COPD } & 5048 & 4590 & 4513\end{array}$

Figure 3 Multivariable-adjusted ORs for COPD according to plasma urate percentiles in the Copenhagen General Population Study (CGPS) and the Copenhagen City Heart Study combined. Solid lines are multivariable-adjusted ORs using a polynomial smoother and dashed lines indicate $95 \% \mathrm{Cl}$ derived from restricted cubic spline regression. Plasma urate percentiles are assigned by sex, study and year of measurement. The analyses were multivariable adjusted for age, sex, smoking status, cumulative smoking, body mass index, physical activity during leisure time, intake of meat (only available for CGPS), intake of alcohol, income, education, pre-existing cardiovascular disease, exposure to occupational dust and fumes, plasma creatinine, plasma C-reactive protein and study as a standard variable. Up to 6809 individuals with self-reported asthma were excluded.

$19 \mu \mathrm{mol} / \mathrm{L}(18.6-19.1)$ higher plasma urate with an $F$ value of 3037 and an $\mathrm{R}^{2}$ of $2.7 \%$ (figure 4 ). Mean plasma urate ranged from $269 \mu \mathrm{mol} / \mathrm{L}(267-270)$ to $346 \mu \mathrm{mol} / \mathrm{L}(345-348)$ in individuals with no urate-increasing and four urate-increasing alleles, respectively. In the combined studies, multivariable-adjusted $100 \mu \mathrm{mol} / \mathrm{L}$ higher plasma urate was associated with $-1.54 \%$ 


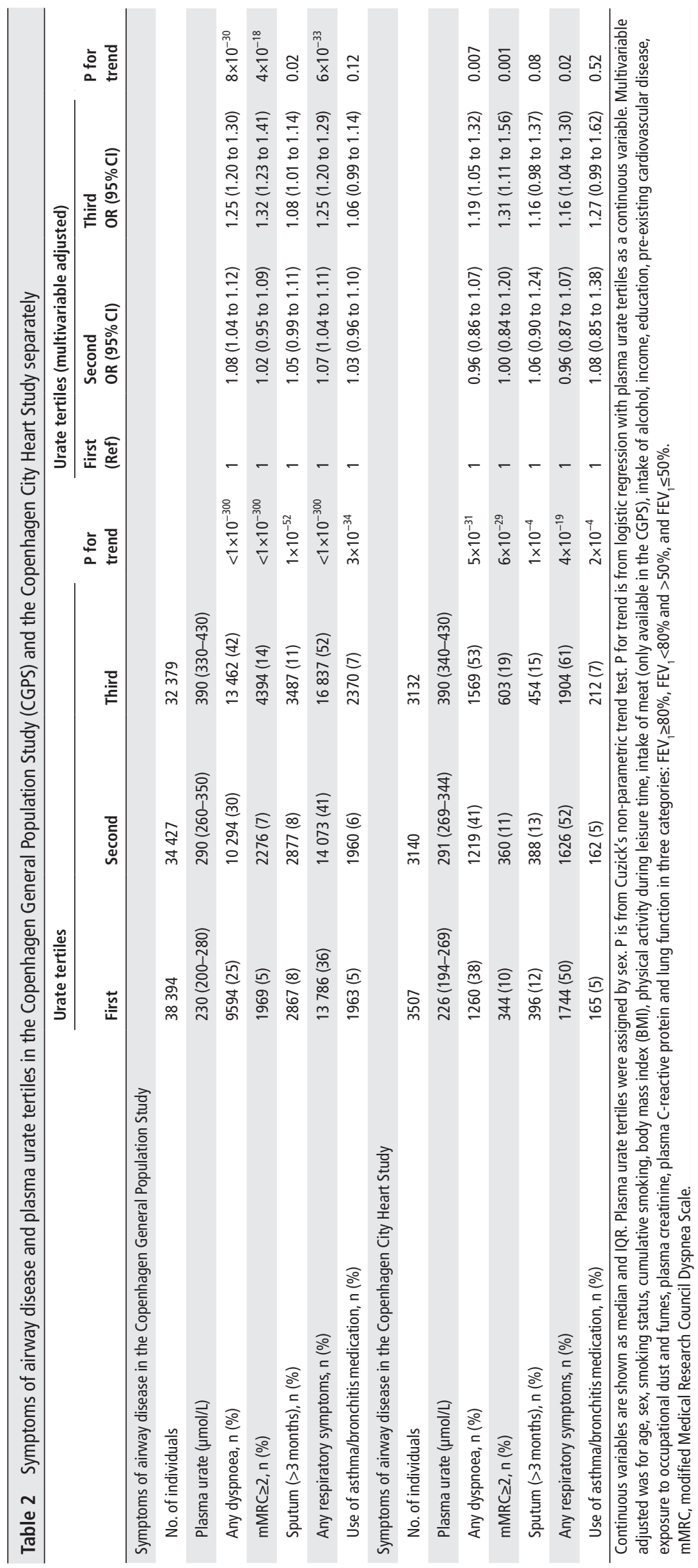

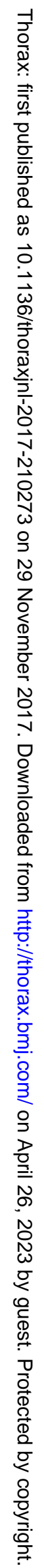




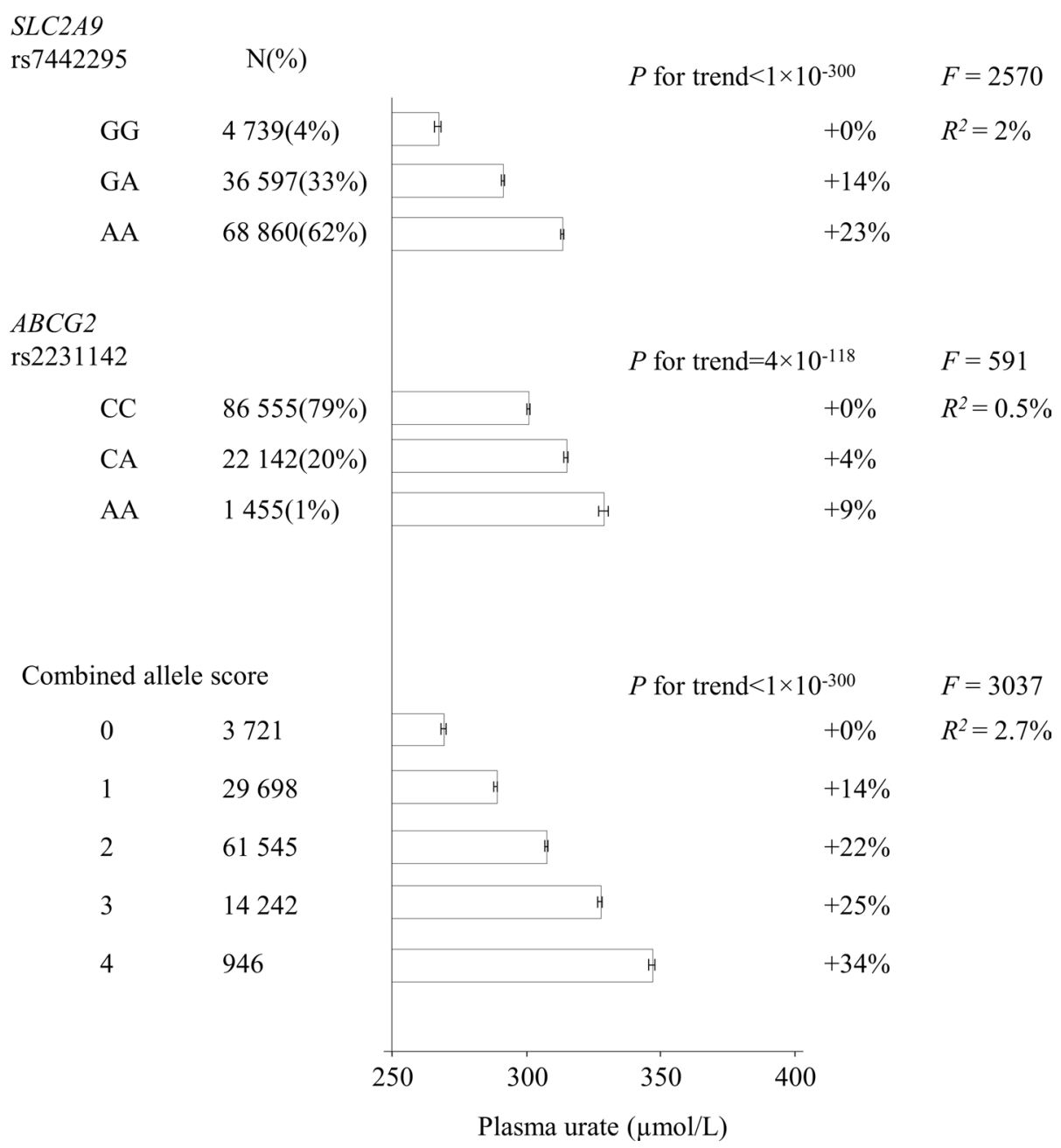

Figure 4 SLC2A9 rs7442295, ABCG2 rs2231142, and the combined allele score and plasma urate in the Copenhagen General Population Study and the Copenhagen City Heart Study combined. Results are means with $95 \% \mathrm{Cls}$. $\mathrm{R}^{2}$ : measure of variation explained by the genotype. F: strength of the instrument. P for trend is from a non-parametric trend test.

(95\% CI -1.67 to -1.40$)$ lower $\mathrm{FEV}_{1} \%$ predicted and $-1.57 \%$ (95\% CI -1.69 to -1.44 ) lower FVC \% predicted; however, the corresponding causal estimates from IV analyses were $-0.46 \%$ (95\% CI -1.17 to 0.25 ) and $-0.40 \%$ (95\% CI -1.03 to 0.23 ) with strong evidence that the observational and genetic estimates differed ( $\mathrm{P}<0.001$ for comparison of estimates) (figure 5). For respiratory symptoms, the observational ORs per $100 \mu \mathrm{mol} / \mathrm{L}$ higher plasma urate were 1.17 (95\% CI 1.15 to 1.20$)$ for any dyspnoea, 1.27 (95\% CI 1.24 to 1.31 ) for severe dyspnoea and 1.17 (95\% CI 1.15 to 1.19 ) for any respiratory symptoms; the corresponding genetic estimates were 1.04 (0.95-1.14), 0.99 $(0.85-1.16)$ and $1.03(0.94-1.12)$, respectively. Stratified on study, the combined allele score was not associated with respiratory symptoms in either of the studies (online supplementary table 6). For COPD, the ORs per $100 \mu \mathrm{mol} / \mathrm{L}$ higher plasma urate were 1.08 (1.06-1.11) observationally and 1.01 (0.88$1.15)$ genetically.

\section{Sensitivity analyses}

We found a moderately stronger association of plasma urate with $\mathrm{FEV}_{1} \%$ predicted among ever-smokers, however only in the CGPS (P for interaction=0.004) (figure 2). We found no interaction of plasma urate with smoking status for FVC \% predicted (online supplementary figure 1) or for the risk of COPD (online supplementary figure 4); however, we found an interaction with smoking status for $\mathrm{FEV}_{1} / \mathrm{FVC}$, although the associations pointed in opposite directions in the two populations (online supplementary figure 2). We found no interaction of the combined allele score with smoking or sex on $\mathrm{FEV}_{1} \%$ predicted, FVC \% predicted, respiratory symptoms or COPD. Within the 14151 individuals with COPD, we found similar associations between plasma urate and $\mathrm{FEV}_{1} \%$ predicted and $\mathrm{FVC} \%$ predicted, as well as symptoms of airway disease (online supplementary figures 5 and 6 and supplementary table 7), as in the total population (figure 2, table 2 and online supplementary figure 1). However, among this subgroup, we found no interaction of plasma urate with smoking status on lung function measurements. Furthermore, all results were similar in a complete case population where all individuals with missing values in one or several covariates had been excluded (online supplementary figures 7 and 8 and supplementary table 8). Finally, when using study as a cluster variable instead of as a standard variable the results were similar (online supplementary figures 9-12).

\section{DISCUSSION}

In this study of 114979 individuals from two independent general population studies, we found that, in observational analyses, higher plasma urate was modestly associated with lower $\mathrm{FEV}_{1} \%$ predicted, lower FVC \% predicted and higher risk of symptoms of airway disease as well as COPD. This contrasts with 


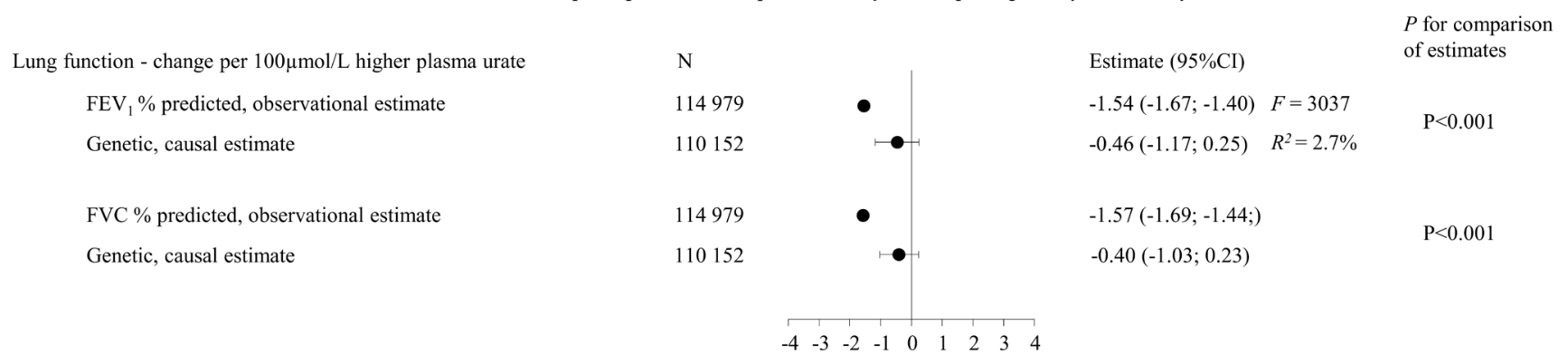

Respiratory symptoms - risk per $100 \mu \mathrm{mol} / \mathrm{L}$ higher plasma urate Any dyspnoea, observational estimate Genetic, causal estimate Severe dyspnoea, observational estimate Genetic, causal estimate Any respiratory symptoms, observational estimate Genetic, causal estimate
114979 110152 114979 110152 114979 110152
$\mathrm{P}<0.001$

$\mathrm{P}<0.001$

$\mathrm{P}<0.001$ $\begin{array}{lll}1.2 & 1.4 & 1.6\end{array}$

Odds ratio
Chronic obstructive pulmonary disease - risk per $100 \mu \mathrm{mol} / \mathrm{L}$ higher plasma urate

Observational estimate

Causal estimate
108170

103628

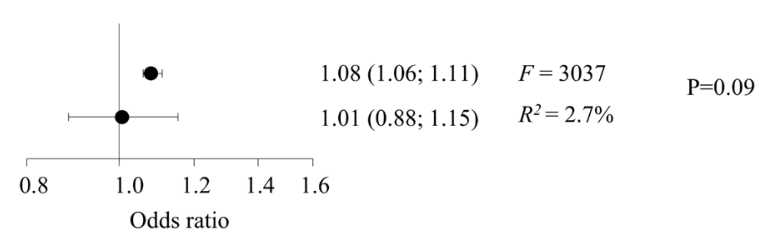

Figure 5 Observational and causal, genetic estimates for lung function, risk of respiratory symptoms and risk of COPD for a $100 \mu \mathrm{mol} / \mathrm{L}$ higher plasma urate in the Copenhagen General Population Study and Copenhagen City Heart Study combined. Observational estimates are multivariable adjusted which includes adjusting for study as a standard variable. Severe dyspnoea was defined as modified Medical Research Council (mMRC) $\geq 2$. Genetic, causal estimate is an OR obtained by using the control function estimator which yields ORs for genetically determined increases in plasma urate, and Cls are estimated using bootstrap with bias correction. $F$-statistics evaluates the strength of the instrument. $\mathrm{R}^{2}$ : measure of variation explained by the allele score. P for comparison of estimates is from Hausman's test comparing the observational and genetic estimates.

the a priori assumption that plasma urate, as a powerful antioxidant, is positively associated with lung function. However, the Mendelian randomisation study did not show an association between genetically high plasma urate and $\mathrm{FEV}_{1} \%$ predicted, FVC \% predicted, symptoms of airway disease or COPD. Thus, our data do not support a causal relationship between higher plasma urate per se and lung function impairment.

Several possible mechanisms may explain this discrepancy between the observational and genetic analyses. First, the observational associations may be explained by other common factors that are associated with both high plasma urate and low lung function. High plasma urate has previously been associated with both BMI and cardiovascular disease, ${ }^{31}$ and although we adjusted for these, unrecognised cardiovascular disease, other chronic illnesses with high purine turnover, or other unmeasured lifestyle factors could still confound the associations between plasma urate and lung function and COPD. Second, the observational associations could be due to reverse causation; pulmonary hypoxia may promote purine catabolism, ${ }^{32}$ which could lead to increased production of urate. Third, one could speculate that high xanthine oxidase activity could lead to simultaneous high plasma urate and lung tissue damage from oxidative stress. Xanthine oxidase catalyses the oxidation of xanthine and hypoxanthine into plasma urate and superoxide and is found in the liver ${ }^{33}$ as well as in epithelial lining fluid of the respiratory tract. ${ }^{34}$ Since superoxide is a free radical with the potential to cause oxidative damage to biological molecules, one could observe high urate with simultaneous high oxidative stress in vivo.

Previous results from observational studies have been inconsistent. Whereas two studies found high plasma urate to be associated with low lung function, ${ }^{15} 17$ and more severe dyspnoea, ${ }^{17}$ another study found no association with dyspnoea, exacerbations or spirometry in patients with COPD. ${ }^{14}$ In contrast, in a study of smoking patients with COPD, plasma urate was lower compared with levels in smokers with normal lung function, ${ }^{16}$ and in a 
cohort of $>200000$ individuals, a weak association was found between high urate and lower incidence of COPD, but with a stronger association in current smokers. ${ }^{18}$ In the present study, we used a novel approach to address the question of whether the association between plasma urate per se and lung function is causal in a large general population setting, and found no support for causality. However, a plasma urate measurement may still provide valuable information to the clinician on the risk of low lung function additional to information from smoking and other relevant socioeconomic factors.

Strengths of our study include the use of two independent cohorts and two different designs; an observational and a genetic design. The latter allows us to assess the causal effect of high plasma urate per se on lung function, respiratory symptoms and risk of COPD, free of confounding and reverse causation. The SLC2A9 rs7442295 and ABCG2 rs2231142 genotypes seem like valid instruments for urate because (1) they are robustly associated with plasma urate, combined they explain $2.7 \%$ of the variation in plasma urate, and has a very large $F$ value of 3037 ; (2) the genes encode transporters involved in renal urate reabsorption and excretion, and likely only assert their effects through plasma urate levels; and (3) we show that both separately and combined they are not associated with any of the measured potential confounding factors for the association of plasma urate with lung function, respiratory symptoms and COPD. Other strengths include a large sample size from a homogenous general population, and adjustment for a large number of potential confounders in the observational analyses.

Some limitations should also be considered. First, as all individuals were of Danish descent, our results may not necessarily apply to other ethnicities; however, this minimises the risk of population stratification affecting genetic analyses and we are not aware of data to suggest that the present results should not be applicable to most ethnic groups. Second, plasma urate and epithelial lining fluid urate are not well correlated, ${ }^{2}$ and it is possible that urate levels in epithelial lining fluid play a different or more significant role in protection against lung oxidative stress from, for example, air pollution than plasma urate levels. Third, we did not adjust $\mathrm{P}$ values for multiple testing; however, as the association between plasma urate and lung function impairment was robust, although it does not seem to be causally related, play of chance seems unlikely. Finally, although we did not lack power in the Mendelian randomisation analyses of the continuous outcomes (we would need 43000 individuals to obtain $80 \%$ power to show a difference of at least $0.02 \%$ lower $\mathrm{FEV}_{1} \%$ predicted or FVC \% predicted at two-sided $\mathrm{P}<0.05$ ), the analysis of our binary outcome did have low power, and our minimum detectable OR with $80 \%$ power at two-sided $\mathrm{P}<0.05$ and 14000 COPD cases was 1.16 . $^{35}$ Thus, we cannot, from the present study, exclude a very small causal effect of plasma urate on risk of COPD.

In conclusion, contrary to that hypothesised, we found that higher plasma urate was associated with lower lung function and higher risk of respiratory symptoms and COPD in observational analyses; however, genetically higher plasma urate was not associated with any of these outcomes. Thus, our data do not support a causal relationship between higher plasma urate per se and lower lung function or higher risk of COPD.

Contributors CJK, SVK, SA, SFN and BGN designed the research. CJK analysed the data. CJK, SVK, SA, SFN and BGN interpreted the data and wrote the manuscript. BGN had the primary responsibility for final content. All authors had full access to all the data including statistical reports and tables, have read and approved the final manuscript, and take responsibility for the integrity and accuracy of the data analysis.
Funding This work was supported by the Michaelsen Foundation, Copenhagen, Denmark, the Danish Council for Independent Research, Medical Sciences, Copenhagen, Denmark, and Chief Physician Johan Boserup and Lise Boserup Foundation, Haslev, Denmark.

Disclaimer The funders had no role in design, collection, analysis or interpretation of data, or in the decision to submit for publication.

Competing interests None declared.

Ethics approval The studies were approved by Herlev and Gentofte Hospital and Danish Ethical Committees.

Provenance and peer review Not commissioned; externally peer reviewed.

(c) Article author(s) (or their employer(s) unless otherwise stated in the text of the article) 2018. All rights reserved. No commercial use is permitted unless otherwise expressly granted.

\section{REFERENCES}

1 Peden $\mathrm{DB}$, Hohman $\mathrm{R}$, Brown ME, et al. Uric acid is a major antioxidant in human nasal airway secretions. Proc Natl Acad Sci U S A 1990;87:7638-42.

2 van der Vliet $A, O^{\prime}$ Neill CA, Cross CE, et al. Determination of low-molecular-mass antioxidant concentrations in human respiratory tract lining fluids. Am J Physiol 1999;276:L289-L296.

3 Becker BF. Towards the physiological function of uric acid. Free Radic Biol Med 1993;14:615-31.

4 Eiserich JP, van der Vliet A, Handelman GJ, et al. Dietary antioxidants and cigarette smoke-induced biomolecular damage: a complex interaction. Am J Clin Nutr 1995:62:1490S-500.

5 Kelly FJ, Tetley TD. Nitrogen dioxide depletes uric acid and ascorbic acid but not glutathione from lung lining fluid. Biochem J 1997;325(Pt 1):95-9.

6 Rahman I, Biswas SK, Kode A. Oxidant and antioxidant balance in the airways and airway diseases. Eur I Pharmacol 2006;533:222-39.

7 Uchida K, Shiraishi M, Naito Y, et al. Activation of stress signaling pathways by the end product of lipid peroxidation. 4-hydroxy-2-nonenal is a potential inducer of intracellular peroxide production. J Biol Chem 1999;274:2234-42.

8 Kirkham PA, Spooner G, Ffoulkes-Jones C, et al. Cigarette smoke triggers macrophage adhesion and activation: role of lipid peroxidation products and scavenger receptor. Free Radic Biol Med 2003;35:697-710.

9 Carp H, Miller F, Hoidal JR, et al. Potential mechanism of emphysema: alpha 1-proteinase inhibitor recovered from lungs of cigarette smokers contains oxidized methionine and has decreased elastase inhibitory capacity. Proc Natl Acad Sci U S A 1982;79:2041-5.

10 McNeil JD, Wiebkin OW, Betts WH, et al. Depolymerisation products of hyaluronic acid after exposure to oxygen-derived free radicals. Ann Rheum Dis 1985;44:780-9.

11 Uchiyama H, Dobashi Y, Ohkouchi K, et al. Chemical change involved in the oxidative reductive depolymerization of hyaluronic acid. I Biol Chem 1990:265:7753-9.

12 McKee CM, Penno MB, Cowman M, et al. Hyaluronan (HA) fragments induce chemokine gene expression in alveolar macrophages. The role of HA size and CD44. I Clin Invest 1996;98:2403-13.

13 Wang RD, Tai H, Xie C, et al. Cigarette smoke produces airway wall remodeling in rat tracheal explants. Am J Respir Crit Care Med 2003;168:1232-6.

14 Garcia-Pachon E, Padilla-Navas I, Shum C. Serum uric acid to creatinine ratio in patients with chronic obstructive pulmonary disease. Lung 2007;185:21-4

15 Aida Y, Shibata Y, Osaka D, et al. The relationship between serum uric acid and spirometric values in participants in a health check: the Takahata study. Int I Med Sci 2011;8:470-8.

16 Nicks ME, O'Brien MM, Bowler RP. Plasma antioxidants are associated with impaired lung function and COPD exacerbations in smokers. COPD 2011:8:264-9.

17 Bartziokas K, Papaioannou Al, Loukides S, et al. Serum uric acid as a predictor of mortality and future exacerbations of COPD. Eur Respir J 2014;43:43-53.

18 Horsfall LJ, Nazareth I, Petersen I. Serum uric acid and the risk of respiratory disease: a population-based cohort study. Thorax 2014;69:1021-6.

19 Lawlor DA, Harbord RM, Sterne JA, et al. Mendelian randomization: using genes as instruments for making causal inferences in epidemiology. Stat Med 2008;27:1133-63.

20 Mandal AK, Mount DB. The molecular physiology of uric acid homeostasis. Annu Rev Physiol 2015;77:323-45.

21 Caulfield MJ, Munroe PB, O'Neill D, et al. SLC2A9 is a high-capacity urate transporter in humans. PLoS Med 2008;5:e197.

22 Vitart V, Rudan I, Hayward C, et al. SLC2A9 is a newly identified urate transporter influencing serum urate concentration, urate excretion and gout. Nat Genet 2008:40:437-42.

23 Döring A, Gieger C, Mehta D, et al. SLC2A9 influences uric acid concentrations with pronounced sex-specific effects. Nat Genet 2008;40:430-6. 
24 Wallace C, Newhouse SJ, Braund P, et al. Genome-wide association study identifies genes for biomarkers of cardiovascular disease: serum urate and dyslipidemia. Am J Hum Genet 2008;82:139-49.

25 Woodward OM, Köttgen A, Coresh J, et al. Identification of a urate transporter, ABCG2, with a common functional polymorphism causing gout. Proc Natl Acad Sci U SA 2009;106:10338-42.

26 Nordestgaard BG, Benn M, Schnohr P, et al. Nonfasting triglycerides and risk of myocardial infarction, ischemic heart disease, and death in men and women. JAMA 2007;298:299-308.

27 Løkke A, Marott JL, Mortensen J, et al. New Danish reference values for spirometry. Clin Respir J 2013;7:153-67.

28 Thomsen M, Ingebrigtsen TS, Marott JL, et al. Inflammatory biomarkers and exacerbations in chronic obstructive pulmonary disease. JAMA 2013;309:2353-61.

29 Durrleman S, Simon R. Flexible regression models with cubic splines. Stat Med 1989;8:551-61.
30 Palmer TM, Sterne JA, Harbord RM, et al. Instrumental variable estimation of causal risk ratios and causal odds ratios in Mendelian randomization analyses. Am J Epidemiol 2011;173:1392-403.

31 Palmer TM, Nordestgaard BG, Benn M, et al. Association of plasma uric acid with ischaemic heart disease and blood pressure: mendelian randomisation analysis of two large cohorts. BMJ 2013;347:f4262.

32 Saito H, Nishimura M, Shibuya E, et al. Tissue hypoxia in sleep apnea syndrome assessed by uric acid and adenosine. Chest 2002;122:1686-94.

33 Saksela M, Lapatto R, Raivio KO. Xanthine oxidoreductase gene expression and enzyme activity in developing human tissues. Biol Neonate 1998;74:274-80

34 Pinamonti S, Muzzoli M, Chicca MC, et al. Xanthine oxidase activity in bronchoalveolar lavage fluid from patients with chronic obstructive pulmonary disease. Free Radic Biol Med 1996;21:147-55.

35 Brion MJ, Shakhbazov K, Visscher PM. Calculating statistical power in Mendelian randomization studies. Int J Epidemiol 2013;42:1497-501. 